\title{
Quantum Key Distribution with Classical Bob
}

\author{
Michel Boyer ${ }^{1}$, Dan Kenigsberg ${ }^{2}$ and Tal Mor ${ }^{2}$ \\ 1. Département IRO, Université de Montréal Montréal (Québec) H3C 3J7 CANADA
}

2. Computer Science Department, Technion, Haifa 32000 ISRAEL

(Dated: September 24, 2018)

\begin{abstract}
Secure key distribution among two remote parties is impossible when both are classical, unless some unproven (and arguably unrealistic) computation-complexity assumptions are made, such as the difficulty of factorizing large numbers. On the other hand, a secure key distribution is possible when both parties are quantum. What is possible when only one party (Alice) is quantum, yet the other (Bob) has only classical capabilities? We present a protocol with this constraint, and prove its robustness against attacks: we prove that any attempt of an adversary to obtain information (and even a tiny amount of information) necessarily induces some errors that the legitimate users could notice.
\end{abstract}

Introduction. Processing information using quantum twolevel systems (qubits), instead of classical two-state systems (bits), has lead to many striking results such as the teleportation of unknown quantum states and quantum algorithms that are exponentially faster than their known classical counterpart. Given a quantum computer, Shor's factoring algorithm would render many of the currently used encryption protocols completely insecure, but as a countermeasure, quantum information processing has also given quantum cryptography. Quantum key distribution was invented by Bennett and Brassard (BB84), to provide a new type of solution to one of the most important cryptographic problems: the transmission of secret messages. A key distributed via quantum cryptography techniques can be secure even against an eavesdropper with unlimited computing power, and the security is guaranteed forever.

The conventional setting is as follows: Alice and Bob have labs that are perfectly secure, they use qubits for their quantum communication, and they have access to a classical communication channel which can be heard, but cannot be jammed (i.e. cannot be tampered with) by the eavesdropper. The last assumption can easily be justified if Alice and Bob can broadcast messages, or if they already share some small number of secret bits in advance, to authenticate the classical channel.

In the well-known BB84 protocol as well as in all other suggested protocols, both Alice and Bob perform quantum operations on their qubits (or on their quantum systems). Here we present, for the first time, a protocol in which one party (Bob) is classical. For our purposes, any two orthogonal states of the quantum two-level system can be chosen to be the computational basis $|0\rangle$ and $|1\rangle$. For reasons that will soon become clear, we shall now call the computational basis "classical" and we shall use the classical notations $\{0,1\}$ to describe the two quantum states $\{|0\rangle,|1\rangle\}$ defining this basis. In the protocol we present, a quantum channel travels from Alice's lab to the outside world and back to her lab. Bob can access a segment of the channel, and whenever a qubit passes through that segment Bob can either let it go undisturbed or (1).- measure the qubit in the classical $\{0,1\}$ basis, and (2).- prepare a (fresh) qubit in the classical basis, and send it. If all parties were limited to performing only operations (1) and (2), or doing nothing, they would always be working with qubits in the classical basis, and could never obtain any quantum superposition of the computational-basis states; the qubits can then be considered "classical bits"; the resulting protocol would then be equivalent to a fully classical protocol, and therefore, the operations themselves shall here be considered classical. We thus term this protocol "QKD protocol with classical Bob". One might use the name SemiQuantum Key Distribution (SQKD), since only one party performs operations beyond the above.

The question of how "quantum" a protocol should be in order to achieve a significant advantage over all classical protocols is of great interest. For example, [1, 2, 3, 4] discuss whether entanglement is necessary for quantum computation, [5] shows nonlocality without entanglement, and [6, 7] discuss how much of the information carried by various quantum states is actually classical. We extend this discussion into another domain: quantum cryptography. Such partiallyquantum or semi-quantum protocols of various types might even have advantages over fully quantum protocols, if they are easier to implement in practice. For instance, NMR quantum computing is among the most successful implementations of quantum computing devices while the performed NMR experiments were proven to use no entanglement [1]. Whether SQKD could also have potential practical advantages or not is left for future research.

To define our protocol we follow the definition (see for instance [8]) of the most standard QKD protocol, BB84. The BB84 protocol consists of two major parts: a first part that is aimed at creating a sifted key, and a second (fully classical) part aimed at extracting an error-free, secure, final key from the sifted key. In the first part of BB84, Alice randomly selects a binary value and randomly selects in which basis to send it to Bob, either the computational (" $Z$ ") basis $\{|0\rangle,|1\rangle\}$, or the Hadamard (" $X$ ") basis $\{|+\rangle,|-\rangle\}$. Bob measures each qubit in either basis at random. An equivalent description is obtained if Alice and Bob use only the classical operations (1) and (2) above and the Hadamard [14] quantum gate H. After all qubits have been sent and measured, Alice and Bob publish which bases they used. For approximately half of the qubits Alice and Bob used mismatching bases and these qubits are discarded. The values of the rest of the bits make the sifted key. The sifted key is identical in Alice's and Bob's hands 
if the protocol is error-free and if there is no eavesdropper (known as Eve) trying to learn the shared bits or some function of them. In the second part Alice and Bob use some of the bits of the sifted key (the TEST bits) to test the error-rate, and if it is below some pre-agreed threshold, they select an INFO string from the rest of the sifted key. Finally, an error correcting code (ECC) is used to correct the errors on the INFO string (the INFO bits), and privacy amplification (PA) is used to derive a shorter but unconditionally secure final key from these INFO bits. At that point we would like to mention a key feature relevant to our protocol: it is sufficient to use qubits in just one basis, $Z$, for generating the INFO string, while the other basis is used only for finding the actions of an adversary [13].

A conventional measure of security is the information Eve can obtain on the final key, and a security proof usually calculates (or puts bounds on) this information. The strongest (most general) attacks allowed by quantum mechanics are called joint attacks. These attacks are aimed to learn something about the final (secret) key directly, by using a probe through which all qubits pass, and by measuring the probe after all classical information becomes public. Security against all joint attacks is considered as "unconditional security". The security of BB84 (with perfect qubits sent from Alice to Bob) against all joint attacks was first proven in [8, 9, 10] via various techniques.

Robustness. An important step in studying security is a proof of robustness; see for instance [11] for robustness proof of their entanglement-based protocol, and [12] for suggesting a protocol secure against the photon-number-splitting (PNS) attack, and for proving its robustness. Robustness of a protocol means that any adversarial attempt to learn some information on the key necessarily induces some disturbance. It is a special case, in zero noise, of the more general "information versus disturbance" measure which provides explicit bound on the information available to Eve as a function of the induced error. Robustness also generalizes the no-cloning theorem: while the no-cloning theorem states that a state cannot be cloned, robustness means that any attempt to make an imprint of a state (even an extremely weak imprint) necessarily disturbs the quantum state.

Definitions: A protocol is said to be completely robust if nonzero information acquired by Eve on the INFO string (before Alice and Bob perform the ECC step) implies nonzero probability that the legitimate participants find errors on the bits tested by the protocol. A protocol is said to be completely nonrobust if Eve can acquire the INFO string without inducing any error on the bits tested by the protocol. A protocol is said to be partly robust if Eve can acquire some limited information on the INFO string without inducing any error on the bits tested by the protocol.

Partly-robust protocols could still be secure, yet completely nonrobust protocols are automatically proven insecure $(\mathrm{Cf}$. Fig. 11. As one example, BB84 is fully robust when qubits are

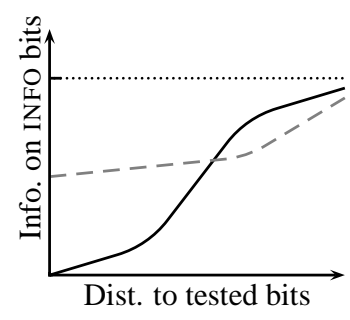

(a)

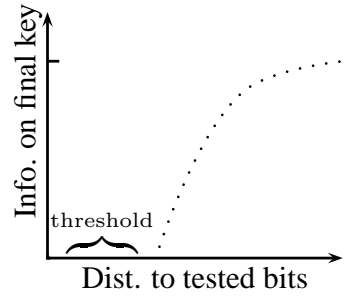

(b)
FIG. 1: (a) Eve's maximum (over all attacks) information on the INFO string vs. the allowed disturbance on the bits tested by Alice and Bob, in a completely robust (solid line), partly robust (dashed), and completely nonrobust (densely dotted) protocol. (b) Robustness should not be confused with security; Eve's maximum information on the final key vs. allowed disturbance in a secure protocol; such a protocol could be completely or partly robust.

used by Alice and Bob but it is only partly robust if photon pulses are used and sometimes two-photon pulses are sent.

Here we prove that our protocol for "quantum key distribution with classical Bob" is completely robust. Another protocol and a proof of its robustness are omitted for the sake of brevity, and will be provided in a future work.

A mock protocol and its complete nonrobustness. Consider the following mock protocol: Alice generates a random qubit in the $Z$-basis. She chooses randomly whether to do nothing, or apply Hadamard gate to transform the qubit to the $X$-basis. Bob flips a coin to decide whether to measure Alice's qubit in the Z-basis (to "SIFT" it) or to reflect it back ("CTRL"), without causing any modification to the information carrier. In case Alice chose $Z$ and Bob decided to SIFT, i.e. to measure in the $Z$ basis, they share a random bit that we call SIFT bit (that may, or may not, be confidential). In case Bob chose CTRL, Alice can check if the qubit returned unchanged, by measuring it in the basis she sent it. In case Bob chose to SIFT and Alice chose the $X$ basis, they discard that bit. The above iteration is repeated for a predefined number of times. At the end of the quantum part of the protocol Alice and Bob share, with high probability, a considerable amount of SIFT bits (also known as the "sifted key"). In order to make sure that Eve cannot gain much information by measuring (and resending) all qubits in the $Z$ basis, Alice can check whether they have a low-enough level of discrepancy on the $X$-basis CTRL bits. In order to make sure that their sifted key is reliable, Alice and Bob must sacrifice a random subset of the SIFT bits, which we denote as TEST bits, and remain with a string of bits which we call INFO bits (INFO and TEST are common in QKD, e.g., in BB84 as previously described).

By comparing the value of the TEST bits, Alice and Bob can estimate the error rate on the INFO bits. If the error rate on the INFO bits is sufficiently small, they use an appropriate Error Correction Code (ECC) in order to correct the errors. If the error rate on the $X$-basis CTRL bits is sufficiently small, Alice and Bob can bound Eve's information, and use an appropriate Privacy Amplification (PA) in order to obtain any desired level of privacy.

At first glance, this protocol may look like a nice way to 
transfer a secret bit from quantum Alice to classical Bob: It is probably resistant to opaque (intercept-resend) attacks, and probably also against all collective attacks (where Eve uses a different probe in each access to each qubit). However, it is completely non-robust; Eve could learn all bits of the INFO string using a trivial attack that induces no error on the bits tested by Alice and Bob (the TEST and CTRL bits). She would not measure the incoming qubit, but rather perform a cNOT from it into a $\left|0^{E}\right\rangle$ ancilla [15]. If Alice chose $Z$ and Bob decide to SIFT (i.e. measures in the $Z$-basis), she measures her ancilla and obtains an exact copy of their common bit, thus inducing no error on TEST bits and learning the INFO string. If, however, Bob decides on CTRL, i.e. reflects the qubit, Eve would perform another cNOT from the returning qubit into her ancilla. This would reset her ancilla, erase the interaction she performed, and induce no error on CTRL bits, thus removing any chance of her being caught.

Note that in this mock protocol, Bob did not use classical operation (2) at all. In the following section we present a protocol in which Bob always sends a qubit to Alice (making use of operation (2) when needed). By always returning all qubits he enforces Eve to delete any information she gained, or else some error is potentially induced.

A Semi-Quantum Key Distribution Protocol. The following protocol remedies the above weakness by not letting Eve know which is a SIFT qubit (that can be safely measured in the computational basis) and which is a CTRL qubit (that should be returned to Alice unchanged). The protocol is aimed at creating an $n$-bit INFO string to be used as the seed for an $m$-bit shared secret key.

Let the integer $n$ be the desired length of the INFO string, and let $\delta>0$ be some fixed parameter.

1. Alice generates $N=8 n(1+\delta)$ random qubits in the $Z$ basis. For each of the qubits, she randomly selects whether to apply the Hadamard gate (" $X$ ") or do nothing ("Z").

2. For each qubit arriving, Bob chooses randomly either to reflect it (CTRL) or to measure it in the $Z$ basis and resend it in the same state he found (to SIFT it). Bob sends the first qubit to Alice after receiving the last qubit, in the same order he received them.

3. Alice measures each qubit in the basis she sent it.

4. Alice publishes which were her $Z$ bits and Bob publishes which ones he chose to SIFT.

It is expected that for approximately $N / 4$ bits, Alice used the $Z$ basis for transmitting, and Bob chose to SIFT; these are the SIFT bits, which form the sifted key. For approximately $N / 4$ bits, Alice used the $Z$ basis and Bob chose CTRL; we refer to these bits as $Z$-CTRL. For approximately $N / 4$ bits, Alice used the $X$ basis and Bob chose CTRL; we refer to these bits as $X$-CTRL. The rest of the bits (those sent in the $X$ basis but chosen as SIFT by Bob) are ignored.
5. Alice checks the error-rate on the CTRL bits and if either the $X$ error-rate or the $Z$ error-rate is higher than some predefined threshold $P_{\mathrm{CTRL}}$ the protocol aborts.

6. Alice chooses at random $n$ SIFT bits to be TEST bits. She publishes which are the chosen bits. Bob publishes the value of these TEST bits. Alice checks the error-rate on the TEST bits and if it is higher than some predefined threshold $P_{\text {TEST }}$ the protocol aborts.

The protocol aborts if there are not enough bits to perform Step 6 or Step 7; this happens with exponentially small probability.

7. Alice and Bob select the first $n$ remaining SIFT bits to be used as INFO bits.

8. Alice publishes ECC \& PA data; she and Bob use them to extract the $m$-bit final key from the $n$-bit INFO string.

A Proof of Robustness. We show that Eve cannot obtain information on INFO bits without being detectable.

Modeling the protocol. Each time the protocol is executed, Alice sends to Bob a state $|\phi\rangle$ which is a product of $N$ qubits, each of which is either $|+\rangle,|-\rangle,|0\rangle$ or $|1\rangle$; those qubits are indexed from 1 to $N$. Each of them is either measured by Bob in the $Z$ basis and resent as it was measured, or simply reflected. Let $m=\left\{m_{1}, m_{2} \ldots m_{r}\right\}$ a set of $r<N$ integers $1 \leq m_{1}<m_{2} \ldots<m_{r} \leq N$, describing the qubits chosen by Bob as SIFT. For $i \in\{0,1\}^{N}$, we denote $i_{m}=i_{m_{1}} i_{m_{2}} \ldots i_{m_{r}}$ the substring of $i$ of length $r$ selected by the positions in $m$; of course $\left|i_{m}\right\rangle=\left|i_{m_{1}} i_{m_{2}} \ldots i_{m_{r}}\right\rangle$.

In the protocol, it is assumed that Bob has no quantum register; he measures the qubits as they come in. The physics would however be exactly the same if Bob used a quantum register of $r$ qubits initialized in state $\left|0^{B}\right\rangle=\left|0^{r}\right\rangle(r$ qubits equal to 0 ), applied the unitary transform defined by $U_{m}|i\rangle\left|0^{B}\right\rangle=|i\rangle\left|i_{m}\right\rangle$ for $i \in\{0,1\}^{N}$, sent back $|i\rangle$ to Alice and postponed his measurement to be performed on that quantum register $\left|i_{m}\right\rangle$; the qubits indexed by $m$ in $|i\rangle$ are thus automatically both measured and resent, and those not in $m$ simply reflected; the $k$ th qubit sent by Alice is a SIFT bit if $k \in m$ and is either $|0\rangle$ or $|1\rangle$; it is a CTRL bit if $k \notin m$. This physically-equivalent modified protocol simplifies the analysis, and we shall thus model Bob's measurement and resending, or reflection, with $U_{m}$.

Eve's attack. Eve's most general attack is comprised of two unitaries: $U_{E}$ attacking qubits as they go from Alice to Bob and $U_{F}$ as they go back from Bob to Alice, where $U_{E}$ and $U_{F}$ share a common probe space with initial state $\left|0^{E}\right\rangle$. The shared probe allows Eve to make the attack on the returning qubits depend on knowledge acquired by $U_{E}$ (if Eve does not take advantage of that fact, then the "shared probe" can simply be the composite system comprised of two independent probes). Any attack where Eve would make $U_{F}$ depend on a measurement made after applying $U_{E}$ can be implemented by unitaries $U_{E}$ and $U_{F}$ with controlled gates so as to postpone 
measurements; since we are giving Eve all the power of quantum mechanics, the difficulty of building such a circuit is of no concern.

The final global state. Delaying all measurements allows considering the final global state of the Eve+Alice+Bob system before all measurements. To state $|\phi\rangle$ sent by Alice, Eve attaches the probe $\left|0^{E}\right\rangle$, applies $U_{E}$ to $\left|0^{E}\right\rangle|\phi\rangle$ and sends Bob his part of the system, $N$ qubits. Taking into account Bob's probe $\left|0^{B}\right\rangle$, the global state is now $\left[U_{E} \otimes I_{M}\right]\left|0^{E}\right\rangle|\phi\rangle\left|0^{B}\right\rangle$ where $I_{M}$ is the identity on Bob's probe space. Then, Bob applies $U_{m}$ to his part of the system, which corresponds to applying $I_{E} \otimes U_{m}$ to the previous global state where $I_{E}$ is the identity on Eve's probe space. Eve's attack on the returning qubits corresponds to applying the unitary $U_{F} \otimes I_{M}$ and the final global state is

$$
\left[U_{F} \otimes I_{M}\right]\left[I_{E} \otimes U_{m}\right]\left[U_{E} \otimes I_{M}\right]\left|0^{E}\right\rangle|\phi\rangle\left|0^{B}\right\rangle .
$$

Proposition 1. If $U_{E}$ induces no error on TEST bits, then there are states $\left|E_{i}\right\rangle$ in Eve's probe space such that for all $i \in\{0,1\}^{N}$

$$
U_{E}\left|0^{E}\right\rangle|i\rangle=\left|E_{i}\right\rangle|i\rangle
$$

If, moreover, $\left(U_{E}, U_{F}\right)$ induces no error on CTRL bits, then there are states $\left|F_{i}\right\rangle$ in Eve's probe space such that for all $i \in\{0,1\}^{N}$,

$$
U_{F}\left|E_{i}\right\rangle|i\rangle=\left|F_{i}\right\rangle|i\rangle
$$

Proof. When $U_{E}$ is applied onto the computational basis, $U_{E}\left|0^{E}\right\rangle|i\rangle=\sum_{j}\left|E_{i, j}\right\rangle|j\rangle$. If for some index $k$ there is some $j$ such that $i_{k} \neq j_{k}$ and $\left|E_{i, j}\right\rangle \neq 0$, then by choosing $m$ such that $k \in m$, Bob can detect this as an error on bit $k$. For Eve's attack to be undetectable on TEST bits, $U_{E}$ must thus be such that $U_{E}\left|0^{E}\right\rangle|i\rangle=\left|E_{i, i}\right\rangle|i\rangle$, namely, $\left|E_{i, j}\right\rangle=0$ for any $j \neq i$, and $\left|E_{i}\right\rangle=\left|E_{i, i}\right\rangle$ satisfies Eq. (2). If Alice sent state $|i\rangle$ for $i \in\{0,1\}^{N}$, the global state is then $\left|E_{i}\right\rangle|i\rangle\left|i_{m}\right\rangle$ and $U_{F}\left|E_{i}\right\rangle|i\rangle=\sum_{j}\left|F_{i, j}\right\rangle|j\rangle$. In order for Eve's attack to be undetectable on $Z$-CTRL bits (whose index is not in $m$ ), $U_{F}$ must be such that $U_{F}\left|E_{i}\right\rangle|i\rangle=\left|F_{i, i}\right\rangle|i\rangle$, namely, $\left|F_{i, j}\right\rangle=0$ for any $j \neq i$ and $\left|F_{i}\right\rangle=\left|F_{i, i}\right\rangle$ then satisfies Eq. (3).

Corollary 1. If the attack $\left(U_{E}, U_{F}\right)$ induces no error on TEST and CTRL bits, then (for all $i \in\{0,1\}^{N}$ and all $m$ ) the final global state (1) if $|\phi\rangle=|i\rangle$ is

$$
\left|F_{i}\right\rangle|i\rangle\left|i_{m}\right\rangle
$$

We now show that if Eve's attack is undetectable by Alice and Bob, then Eve's final state $\left|F_{i}\right\rangle$ is independent of the string $i \in\{0,1\}^{N}$. More precisely

Proposition 2. If $\left(U_{E}, U_{F}\right)$ is an attack that induces no error on TEST and CTRL bits, and if $\left|F_{i}\right\rangle$ is given by Eq. (4), then for all $i, i^{\prime} \in\{0,1\}^{N}$

$$
i, i^{\prime} \in\{0,1\}^{N} \quad \Longrightarrow \quad\left|F_{i}\right\rangle=\left|F_{i^{\prime}}\right\rangle .
$$

Proof. Eq. (5) means that any of the $N$ bits of $i \in\{0,1\}^{N}$ can be flipped at will without affecting Eve's final state $\left|F_{i}\right\rangle$. We thus need only prove that for any two bit strings $i, i^{\prime} \in$ $\{0,1\}^{N}$ that differ only on one bit, say bit $k$, the equality $\left|F_{i}\right\rangle=\left|F_{i^{\prime}}\right\rangle$ holds. We assume wlg that $i_{k}=0$ and $i_{k}^{\prime}=1$. If Alice chooses qubit $k$ to be $X$-CTRL and chooses all the other qubits to be those of $i$ and $i^{\prime}$, then this means that the state $|\phi\rangle$ she sends is $\frac{1}{\sqrt{2}}\left[|i\rangle+\left|i^{\prime}\right\rangle\right]$. Assume now that Bob reflects bit $k$, i.e. that $k \notin m$. This implies that $i_{m}=i_{m}^{\prime}$. By Eq. (4) and linearity, the final state is $\frac{1}{\sqrt{2}}\left[\left|F_{i}\right\rangle|i\rangle+\left|F_{i^{\prime}}\right\rangle\left|i^{\prime}\right\rangle\right]\left|i_{m}\right\rangle$. Since we are interested only in Alice's $k$ th qubit, we trace-out all the other qubits in Alice and Bob's hands. The resulting state

$$
\frac{1}{\sqrt{2}}\left[\left|F_{i}\right\rangle|0\rangle+\left|F_{i^{\prime}}\right\rangle|1\rangle\right]
$$

must be such that the probability of Alice measuring $|-\rangle$ is 0 . Replacing $|0\rangle$ and $|1\rangle$ by their value in terms of $|+\rangle$ and $|-\rangle$, state (6) rewrites as $\frac{1}{2}\left[\left|F_{i}\right\rangle+\left|F_{i^{\prime}}\right\rangle\right]|+\rangle+\frac{1}{2}\left[\left|F_{i}\right\rangle-\left|F_{i^{\prime}}\right\rangle\right]|-\rangle$ and the probability of measuring $|-\rangle$ is 0 iff $\frac{1}{2}\left[\left|F_{i}\right\rangle-\left|F_{i^{\prime}}\right\rangle\right]=$ 0 i.e. $\left|F_{i}\right\rangle=\left|F_{i^{\prime}}\right\rangle$.

Theorem 1. The protocol is completely robust: for any attack $\left(U_{E}, U_{F}\right)$ inducing no error on TEST and CTRL bits, Eve's final state is independent of the states $|\phi\rangle$ sent by Alice, and Eve is thus left with no information on the INFO string.

Proof. By Proposition 2, there is a state $\left|F_{\text {final }}\right\rangle$ in Eve's probe space s.t. for all $i \in\{0,1\}^{N}$, Eve's final state $\left|F_{i}\right\rangle=\left|F_{\text {final }}\right\rangle$. If Alice sends any superposition $|\phi\rangle=\sum_{i} c_{i}|i\rangle$ and Bob chooses any set $m$ of bits to be measured (leaving at least one CTRL bit). Using Eq. (4) with $\left|F_{i}\right\rangle=\left|F_{\text {final }}\right\rangle$ for all $i$ and linearity gives $\left|F_{\text {final }}\right\rangle \sum_{i} c_{i}|i\rangle\left|i_{m}\right\rangle$ as the final global state of the system; Eve's probe state $\left|F_{\text {final }}\right\rangle$ is independent of $i_{m}$ and therefore of the SIFT and INFO bits.

Conclusion. We presented a protocol for QKD with one party who performs only classical operations and proved its robustness. We believe that our work sheds light on how much "quantumness" is required in order to perform classicallyimpossible tasks in general, and secret key distribution in particular. This work was partially supported by the Israeli MOD. We thank Moshe Nazarathy for providing the motivation for this research.

[1] S. L. Braunstein, C. M. Caves, R. Jozsa, N. Linden, S. Popescu, and R. Schack, Phys. Rev. Lett. 83, 1054 (1999).

[2] R. Jozsa and N. Linden, Proc. of the Roy. Soc. of London series A 459, 2011 (2003).

[3] E. Biham, G. Brassard, D. Kenigsberg, and T. Mor, Theoretical Computer Science 320, 13 (2004).

[4] D. Kenigsberg, T. Mor, and G. Ratsaby, Quantum Information and Computation 6, 606 (2006). 
[5] C. H. Bennett, D. P. DiVincenzo, C. A. Fuchs, T. Mor, E. Rains, P. W. Shor, J. A. Smolin, and W. K. Wootters, Phys. Rev. A 59, 1070 (1999).

[6] B. Groisman, S. Popescu, and A. Winter, Physical Review A 72, 32317 (2005).

[7] C. A. Fuchs and M. Sasaki, Quantum Information and Computation 3, 377 (2003).

[8] E. Biham, M. Boyer, P. O. Boykin, T. Mor, and V. Roychowdhury, Journal of Cryptology 19, 381 (2006), and first in STOC'00 (ACM Press, New York, 2000), pp. 715-724.

[9] D. Mayers, J. ACM 48, 351 (2001), ISSN 0004-5411.
[10] P. W. Shor and J. Preskill, Phys. Rev. Lett. 85, 441 (2000).

[11] C. H. Bennett, G. Brassard, and N. D. Mermin, Phys. Rev. Lett. pp. 557-559 (1992).

[12] V. Scarani, A. Acín, G. Ribordy, and N. Gisin, Phys. Rev. Lett. 92, 057901 (2004).

[13] T. Mor, Phys. Rev. Lett. 80, 3137 (1998).

[14] $H|0\rangle=|+\rangle ; H|1\rangle=|-\rangle$.

[15] By "cNot from $A$ into $B$ " we mean that $A$ is the control qubit and $B$ is the target, as is commonly called. 